\title{
Microstructure and Optical Characterization of Mid-Wave HgCdTe Grown by MBE under Different Conditions
}

\author{
Xiao-Fang Qiu ${ }^{1,2}$, Sheng-Xi Zhang ${ }^{2}$, Jian Zhang ${ }^{2}$, Yi-Cheng Zhu ${ }^{2}$, Cheng Dou ${ }^{1}$, San-Can Han ${ }^{1}$, Yan Wu ${ }^{2, *}$ \\ and Ping-Ping Chen ${ }^{2, *}$ \\ 1 School of Material Science \& Engineering, University of Shanghai for Science and Technology, \\ Shanghai 200093, China; 182442555@st.usst.edu.cn (X.-F.Q.); 182442525@st.usst.edu.cn (C.D.); \\ schan@usst.edu.cn (S.-C.H.) \\ 2 State Key Laboratory of Infrared Physics, Shanghai Institute of Technical Physics Chinese Academy of \\ Sciences, Shanghai 200083, China; zhangshengxi@mail.sitp.ac.cn (S.-X.Z.); zj2062@mail.ustc.edu.cn (J.Z.); \\ zycheng@mail.ustc.edu.cn (Y.-C.Z.) \\ * Correspondence: Wu_yan@mail.sitp.ac.cn (Y.W.); ppchen@mail.sitp.ac.cn (P.-P.C.)
}

check for updates

Citation: Qiu, X.-F.; Zhang, S.-X.; Zhang, J.; Zhu, Y.-C.; Dou, C.; Han, S.-C.; Wu, Y.; Chen, P.-P. Microstructure and Optical Characterization of Mid-Wave HgCdTe Grown by MBE under Different Conditions. Crystals 2021, 11, 296. https://doi.org/10.3390/ cryst11030296

Academic Editor: Wen Lei

Received: 24 February 2021

Accepted: 13 March 2021

Published: 16 March 2021

Publisher's Note: MDPI stays neutral with regard to jurisdictional claims in published maps and institutional affiliations.

Copyright: (c) 2021 by the authors. Licensee MDPI, Basel, Switzerland. This article is an open access article distributed under the terms and conditions of the Creative Commons Attribution (CC BY) license (https:// creativecommons.org/licenses/by/ $4.0 /)$.

\begin{abstract}
The mid-wave single-crystal HgCdTe (211) films were successfully grown on GaAs (211) B substrates by molecular beam epitaxy (MBE). Microstructure and optical properties of the MBE growth $\mathrm{HgCdTe}$ films grown at different temperatures were characterized by X-ray diffraction, scanning transmission electron microscopy, Raman and photoluminescence. The effects of growth temperature on the crystal quality of $\mathrm{HgCdTe} / \mathrm{CdTe}$ have been studied in detail. The $\mathrm{HgCdTe}$ film grown at the lower temperature of $151{ }^{\circ} \mathrm{C}$ has high crystal quality, the interface is flat and there are no micro twins. While the crystal quality of the $\mathrm{HgCdTe}$ grown at higher temperature of $155^{\circ} \mathrm{C}$ is poor, and there are defects and micro twins at the $\mathrm{HgCdTe} / \mathrm{CdTe}$ interface. The research results demonstrate that the growth temperature significantly affects the crystal quality and optical properties of $\mathrm{HgCdTe}$ films.
\end{abstract}

Keywords: HgCdTe; molecular beam epitaxy; optical properties; defects; interface

\section{Introduction}

Infrared detectors are widely used in the military, industry and other fields. The majority of current commercially available high-performance IR photodetectors are developed using III-V and II-VI semiconductor, such as InGaAs, InSb, HgCdTe, and type-II superlattices [1-3]. The $\mathrm{HgCdTe}$ and $\mathrm{InSb}$ are the main research objects for military and aerospace applications at mid-wave length. The InSb bandgap is not adjustable and is just suitable for medium-wave applications. The development trend of infrared detectors is to possess two-color or multi-color characteristics with large-area arrays that have a high-temperature resolution [4]. The $\mathrm{HgCdTe}$ infrared materials have dominated the highperformance end of the infrared detector market for decades due to their superior device performance, such as a high detectivity and high quantum efficiency [5]. In recent years, the high-operating-temperature (HOT) mid-wave HgCdTe infrared detectors have attracted attention [6,7]. The main problem caused by the HOT detectors is the increased number of defects and greater low-frequency noise. Improving material performance is the key to develop HOT mid-wave infrared detectors. On the other hand, the mid-wave HgCdTe [8,9] has become a promising avalanche photon detector (APD) material, which has important application prospects in three-dimensional Lidar imaging. For APD materials, high-purity HgCdTe with a low defect density is more critical. Therefore, it is necessary to explore high-performance mid-wave $\mathrm{HgCdTe}$ infrared detection materials.

Molecular beam epitaxy (MBE) is one of the most important growth methods to grow HgCdTe single film [10]. The difficulty of MBE growth $\mathrm{HgCdTe}$ is focused primarily on the fluxes and growth temperatures of $\mathrm{Hg}, \mathrm{CdTe}$, and $\mathrm{Te}$, which directly affects the 
crystal quality and the composition of HgCdTe materials [11]. Due to the low adhesion coefficient of $\mathrm{Hg}$, the growth of $\mathrm{HgCdTe}$ material is highly sensitive to temperature. When the substrate temperature or flux fluctuates during the growth process, several defects appear on the film surface [12], such as Hg-rich or Hg-deficiency, stress streaks, and needleshaped defects. These defects affect the crystal quality of $\mathrm{HgCdTe}$ films, which significantly influences the detector performance. Therefore, the substrate temperature and flux are important factors that affect the growth of $\mathrm{HgCdTe}$ films. The growth temperature of the $\mathrm{HgCdTe}$ epilayer is generally controlled to within $\pm 2{ }^{\circ} \mathrm{C}$ [13], which means that the temperature fluctuation of the $\mathrm{HgCdTe}$ epilayer during the growth must be within $\pm 2{ }^{\circ} \mathrm{C}$. Additionally, the temperature of $\mathrm{HgCdTe}$ films changes with its composition and thickness during growth, which greatly increases the difficulty of attaining high-quality $\mathrm{HgCdTe}$.

In this work, we report the crystal quality characterization of MBE-grown $\mathrm{HgCdTe} / \mathrm{GaAs}$ (211) B epilayers using high-energy electron diffractometer (RHEED) and X-ray double diffraction (XRD, Bruker, Karlsruhe, Germany). The microstructural investigation is performed using scanning electron microscopy (SEM, ZEISS, Jena, Germany), atomic force microscopy (AFM, Bruker, Madison, American), and scanning transmission electron microscopy (STEM, FEI Titan Themis, Hillsboro, American). The optical properties are determined using Raman spectroscopy and variable-temperature photoluminescence (PL). Our observations establish that the temperature significantly affects the microstructure and optical properties of the HgCdTe epilayer.

\section{Materials and Methods}

HgCdTe (211) films were grown on GaAs (211) B substrates with molecular beam epitaxy (MBE) system (DCA R450, Turku, Finland). The growth process was monitored in real-time using RHEED. The growth temperature was measured with an infrared thermometer (or pyrometer), which was calibrated with the indium melting temperature $\left(156^{\circ} \mathrm{C}\right)$.

The GaAs (211) substrate was pre-degassed in a pretreatment chamber at $350^{\circ} \mathrm{C}$ for $60 \mathrm{~min}$, and was deoxidized at $590^{\circ} \mathrm{C}$ in the growth chamber. To reduce the lattice mismatch between the $\mathrm{HgCdTe}$ and GaAs, the buffer layer of $\mathrm{ZnTe} / \mathrm{CdTe}$ composite layer [14] was adopted. The growth conditions of the $\mathrm{HgCdTe}$ epitaxial film were determined by optimizing the growth process parameters, and the $\mathrm{ZnTe} / \mathrm{CdTe}$ buffer layer grows at approximately $260^{\circ} \mathrm{C}$. HgCdTe films were grown with solid CdTe sources instead of $\mathrm{Cd}$ and Te sources. Since the intensity of the atomic/molecular beam current depends not only on the temperature of the beam source furnace, but also other influencing factors, such as the shape of the crucible opening and the surface area of the source material. So, it is difficult to get the right values of flux. Therefore, the flux intensity of the source is estimated by the beam equivalent pressure (BEP) measured by the vacuum gauge at the substrate position. The $\mathrm{HgCdTe}$ (211) epilayer grows at $155^{\circ} \mathrm{C}$ and $151^{\circ} \mathrm{C}$ (with deviation at $\pm 1{ }^{\circ} \mathrm{C}$ ), and the beam equivalent pressure (BEP) ratio of $\mathrm{Hg} / \mathrm{Te} / \mathrm{CdTe}$ is approximately 125:1.56:1. The BEP value of the $\mathrm{Hg}$ is about $4.5 \times 10^{-5}$ Torr. The composition is controlled by the growth temperature and BEP ratio, and the growth parameters of the epilayers are shown in Table 1.

Table 1. Growth conditions of the HgCdTe films.

\begin{tabular}{ccccccc}
\hline \multirow{2}{*}{ Sample } & Component $(\mathbf{x})$ & $\begin{array}{c}\text { Thickness } \\
(\mu \mathrm{m})\end{array}$ & \multicolumn{2}{c}{ Growth Temperature $\left({ }^{\circ} \mathbf{C}\right)$} & \multirow{2}{*}{ CdTe Buffer BEP } \\
\cline { 3 - 5 } & & CdTe Buffer & HgCdTe Layer & (Torr) \\
\hline Sample 1 & 0.310 & 3.40 & 260 & 155 & $3.51 \times 10^{-7}$ \\
\hline Sample 2 & 0.298 & 2.97 & 260 & 151 & $3.57 \times 10^{-7}$ \\
\hline
\end{tabular}

The growth process of the $\mathrm{HgCdTe} / \mathrm{GaAs}$ (211) B epilayer is shown in Figure 1. Figure 1a,c are the RHEED images of the CdTe buffer layer for Sample 1 and Sample 2 in the [011] orientation. Figure $1 \mathrm{~b}, \mathrm{~d}$ are the RHEED images of the HgCdTe epilayer of Sample 1 and Sample 
2 in the [011] orientation. The RHEED patterns exhibit sharp stripes at various growth stages, indicating two-dimensional growth of CdTe and HgCdTe single-crystal films on GaAs (211) B substrates.
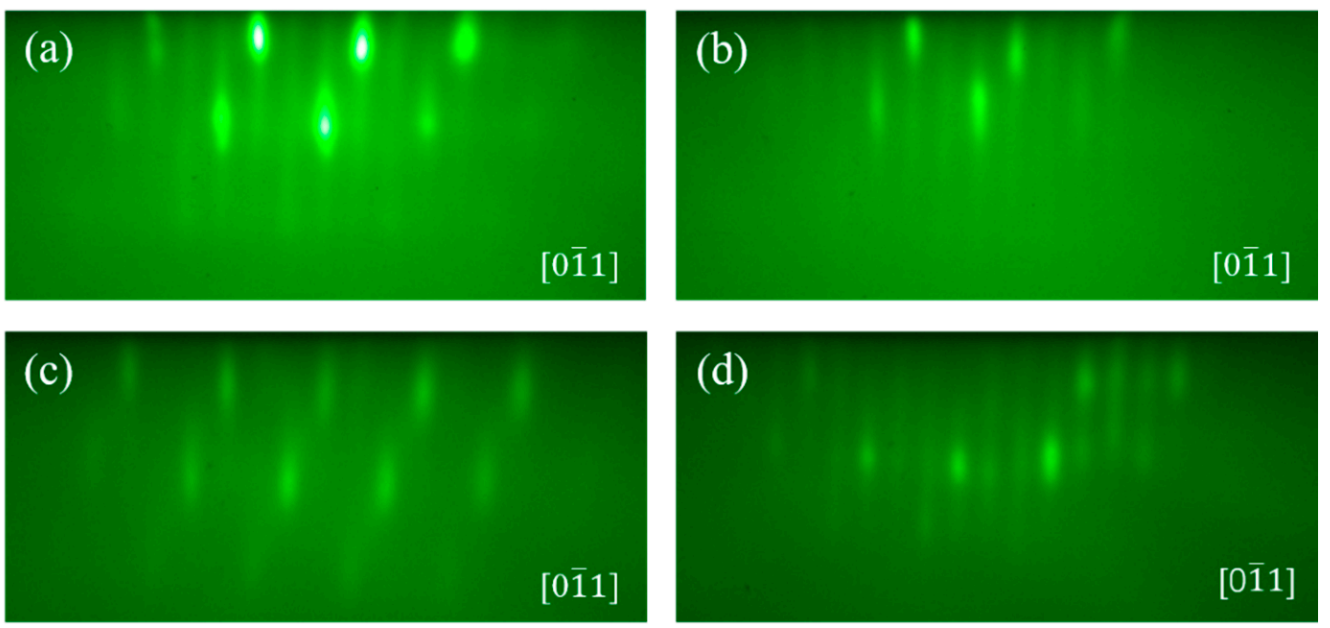

Figure 1. (a,c) RHEED images of the CdTe buffer for Sample 1 and Sample 2, and (b,d) RHEED images before the end of the growth for HgCdTe films (Sample 1 and Sample 2).

Fourier transmission infrared spectroscopy (FTIR) is used to measure the bandgap of the $\mathrm{Hg}_{1-\mathrm{x}} \mathrm{Cd}_{\mathrm{x}} \mathrm{Te}$ epilayer films [15]. The internal humidity of the FTIR device is less than $70 \%$, the resolution is $8 \mathrm{~cm}^{-1}$, and the diaphragm size is $8 \mathrm{~mm}$. The $\mathrm{x}$ component of $\mathrm{Hg}_{1-\mathrm{x}} \mathrm{Cd}_{\mathrm{x}} \mathrm{Te}$ can be calculated from the relationship between $\mathrm{E}_{\mathrm{g}}$ and $\mathrm{x}$ as:

$$
E_{g}(x, T)=-0.295+1.87 x-0.28 x^{2}+\left(6-14 x+3 x^{2}\right) \times 10^{-4} \times T+0.35 x^{4}
$$

The composition $(x=0.310$ and 0.298$)$ can also be measured using photoluminescence (PL), excited with a laser power of $100 \mathrm{~mW}$, a resolution of $16 \mathrm{~cm}^{-1}$ and the size of diaphragm is $8 \mathrm{~mm}$. As shown in Table 2, it can be seen that the fitting results of FTIR and PL match quite well.

Table 2. Composition measured by FTIR/PL.

\begin{tabular}{ccc}
\hline Sample & FTIR & PL \\
\hline Sample 1 & 0.310 & 0.309 \\
Sample 2 & 0.298 & 0.298 \\
\hline
\end{tabular}

\section{Results and Discussion}

\subsection{X-ray Diffraction}

The X-ray diffraction (XRD) was used to characterize the crystal quality and orientation of the HgCdTe (211) film, as shown in Figure 2. The graphs for Sample 1 and Sample 2 have no obvious miscellaneous peaks, as shown in Figure 2a. The diffraction peaks at $71.4^{\circ}$ and $83.5^{\circ}$ correspond to the $\mathrm{HgCdTe}(422)$ and the GaAs (422). The Figure $2 \mathrm{~b}$ shows the HgCdTe (422) XRD rocking curve FWHMs of Sample 1 and Sample 2, which are 179 and 128 arcsec, respectively. This indicates that the crystal quality of Sample 2 is better. 

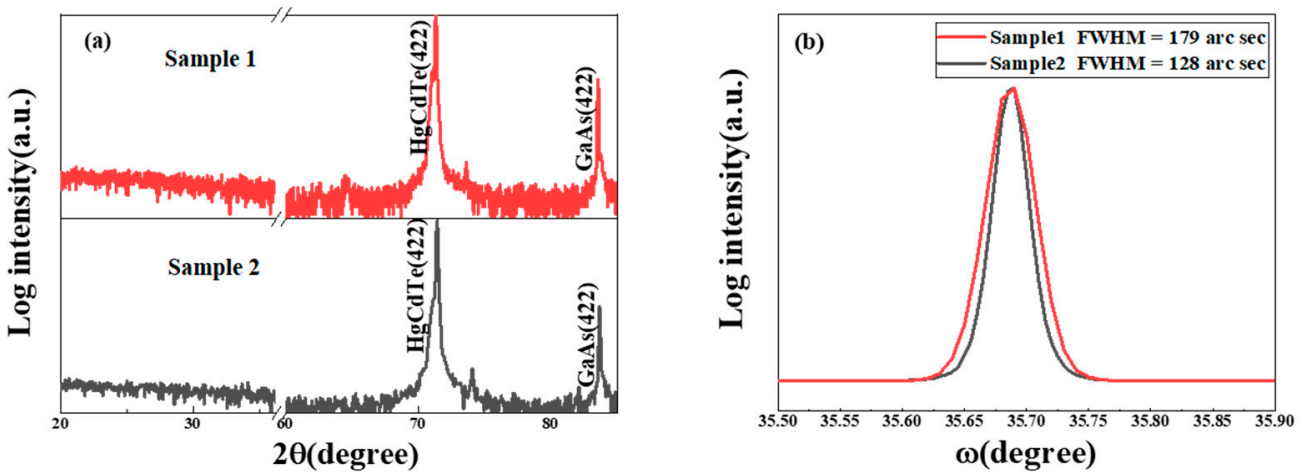

Figure 2. The XRD curve for HgCdTe (422) of Sample 1 and Sample 2: (a) $2 \theta-\omega$ results; (b) HgCdTe (422) XRD rocking curve of Sample 1 and Sample 2.

\subsection{Scanning Electron Microscope}

Figure $3 \mathrm{a}, \mathrm{b}$ are the SEM images of the HgCdTe surface of Sample 1 and Sample 2. Figure 3a is the SEM image of Sample 1. The figure inset is an enlarged image of a surface defect. Larger voids will be formed on the surface at lower $\mathrm{Hg}$ flux or higher growth temperature (as shown in Figure 3a). The size of the voids depends on the degree of $\mathrm{Hg}$ deficiency [13]. As the degree of $\mathrm{Hg}$ deficiency becomes more serious, the $\mathrm{Hg}$-deficiency gradually increases, and the size usually does not exceed $30 \mu \mathrm{m}$. Figure $3 \mathrm{~b}$ shows the surface morphology of Sample 2 without $\mathrm{Hg}$-deficiency. Obviously, the macroscopic defects of $\mathrm{HgCdTe}$ film grown at $151^{\circ} \mathrm{C}$ are less than those of $\mathrm{HgCdTe}$ films grown at $155^{\circ} \mathrm{C}$. The surface quality of $\mathrm{HgCdTe}$ epilayer is very sensitive to the growth conditions owing to the low adhesion coefficient of $\mathrm{Hg}$. Therefore, the growth temperature plays a crucial role in the surface quality of $\mathrm{HgCdTe}$ crystals under the condition of constant BEP ratio.
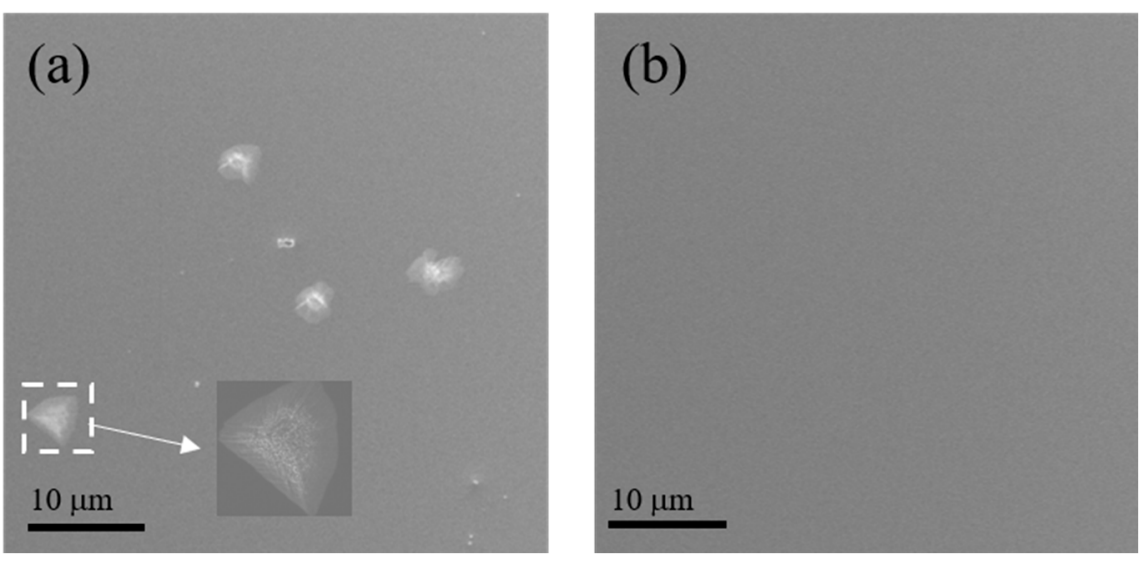

Figure 3. SEM images of the HgCdTe thin films grown at different temperatures of (a) Sample 1 and the inset is an Hg-deficiency enlarged image; (b) Sample 2.

\subsection{Atomic Force Microscopy}

Figure 4 shows the AFM images of HgCdTe surface of Sample 1 (a) and Sample 2 (b). As can be seen from Figure 4, the roughness (RMS values) of both samples is less than $2 \mathrm{~nm}$, indicating the smooth surface of the $\mathrm{HgCdTe}$ films. Meanwhile there are nano-ripples along the [1111] direction on the surface of the both samples, similar with the report [16]. The nano-ripples can be attributed to meandering macro step edges formed due to an instability in the step flow growth mode. Nano-ripples are affected by growth temperature and lattice mismatch between substrate and epilayer. The nano-ripple spacings of Sample 1 and Sample 2 are approximately 0.33 and $0.25 \mu \mathrm{m}$, respectively. The spacing between $\mathrm{HgCdTe}$ nano-ripples of Sample 1 (grown at $151^{\circ} \mathrm{C}$ ) is smaller than that of Sample 2 (grown at $155^{\circ} \mathrm{C}$ ) and the height is lower than that of Sample 2, indicating that the density of $\mathrm{HgCdTe}$ nano-ripples grown at $151{ }^{\circ} \mathrm{C}$ is higher than that of $\mathrm{HgCdTe}$ nano-ripples grown at 
$155^{\circ} \mathrm{C}$. Nano-ripples morphology is affected by the availability of atoms on the surface [17]. The availability of surface atoms is closely related to growth conditions such as growth temperature, so the surface morphology under different growth conditions is different.
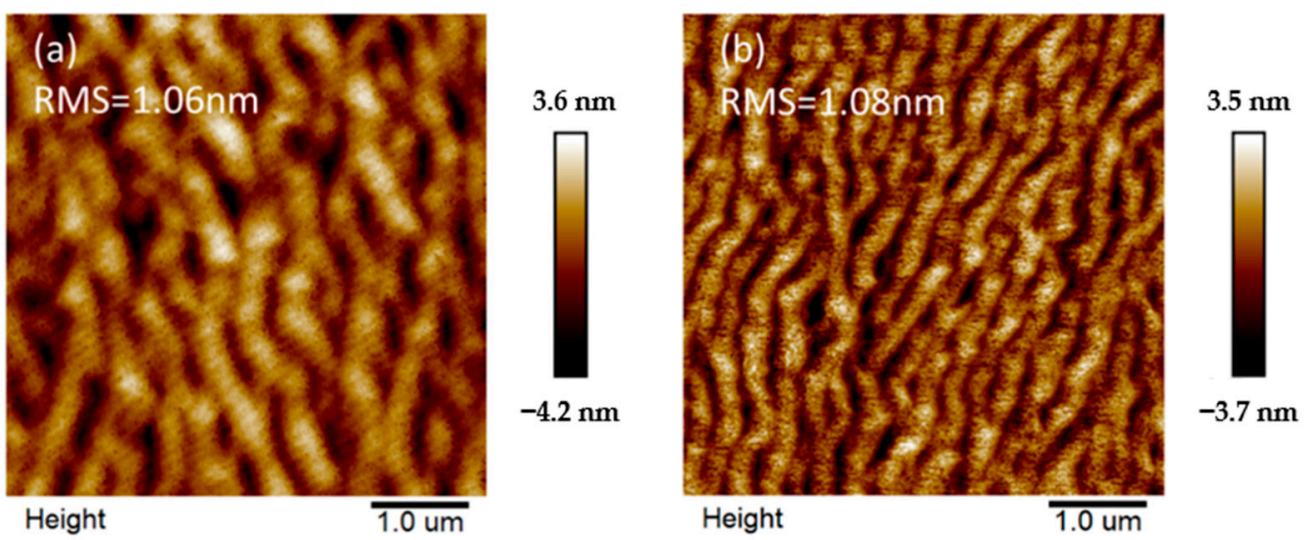

Figure 4. Surface morphology of the $\mathrm{HgCdTe}$ (211) thin films grown at different temperatures of (a) Sample 1 and (b) Sample 2.

\subsection{Scanning Transmission Electron Microscopy}

The interface of the CdTe buffer layer and $\mathrm{HgCdTe}$ epilayer was characterized by scanning transmission electron microscopy (STEM), as shown in Figure 5a-c for Sample 1 and Figure $5 \mathrm{~d}-\mathrm{f}$ for Sample 2. As can be seen from Figure 5a, there is an obvious interface between the $\mathrm{HgCdTe}$ film grown at $155^{\circ} \mathrm{C}$ and $\mathrm{CdTe}$ buffer layer. Figure $5 \mathrm{~d}$ shows that there is no obvious interface between $\mathrm{HgCdTe}$ grown at $151{ }^{\circ} \mathrm{C}$ and $\mathrm{CdTe}$, and no obvious twinning and stacking fault. Figure 5b,e shows the enlarged STEM images of Sample 1 and Sample 2, respectively. As can be seen from Figure 5b, lattice distortion occurs in the epilayer, and stacking fault can be found in the inset. The crystal lattices before and after stacking faults are symmetrical, indicating the presence of twins in the epilayer, while in Figure 5e, no reflection twinning can be seen. Figure 5c shows the fast Fourier transform (FFT) pattern at the interface between $\mathrm{HgCdTe}$ and CdTe of Sample 1. It can be seen that there are two diffraction lattices and the lattice is blurred, which indicates the existence of stacking faults and micro-twinning in the HgCdTe epilayer. Figure $5 \mathrm{f}$ shows the FFT pattern at the interface between $\mathrm{HgCdTe}$ and CdTe of Sample 2. The diffraction spots are clear and bright, indicating that no twinning and stacking faults were found in the $\mathrm{HgCdTe}$ layer grown at $151^{\circ} \mathrm{C}$. It shows that the crystal quality of the $\mathrm{HgCdTe}$ layer grown at $151^{\circ} \mathrm{C}$ is better than that grown at $155^{\circ} \mathrm{C}$.

During crystal growth, the lattice mismatch between $\mathrm{CdTe}$ and $\mathrm{HgCdTe}$ leads to the formation of dislocation at the interface. At the same time, the formation of twinning is greatly affected by temperature. When the growth temperature is $151^{\circ} \mathrm{C}$, there is no large amount of micro-twinning in the $\mathrm{HgCdTe}$ layer at the interface, and the lattice quality at the interface is better. However, twinning appeared at the growth temperature of $155^{\circ} \mathrm{C}$. This due to the occurrence of Te-rich defects at high growth temperature, which leads to stacking faults and the formation of micro-twins.

In Figure 5b, the lamellar growth twins parallel to the (111) plane [18]. Micro-twins tend to appear along the [111] orientation because the surface energy of (111) plane is lower than that of (211) and (331) planes, so the dislocation is easier to migrate to (111) plane [19]. In general, the formation of twinning can be inhibited by the following methods: (1) The lattice matching between the epilayer and the substrate; (2) Adjust the growth direction of the epilayer (between [111] and [211]); (3) Optimize growth conditions to reduce twins, etc. 

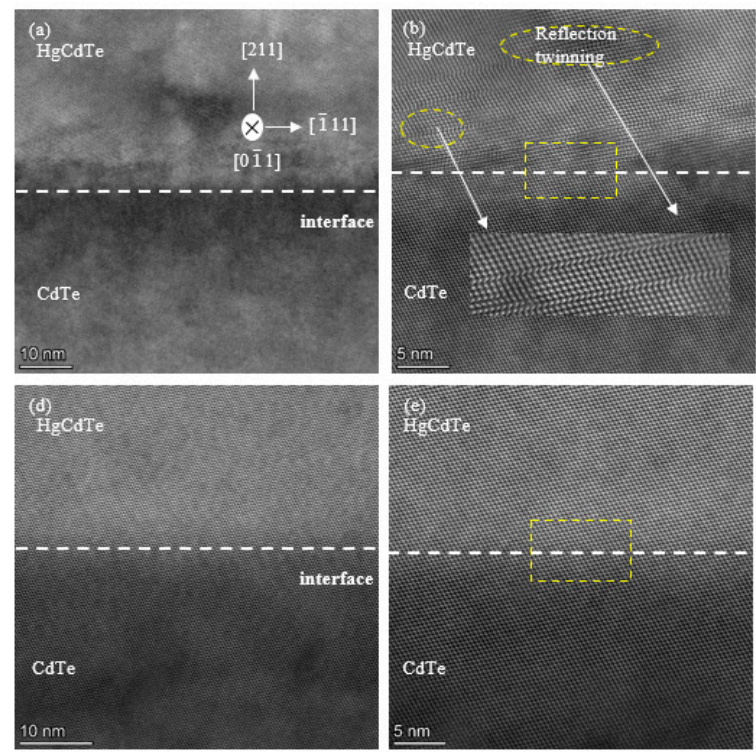
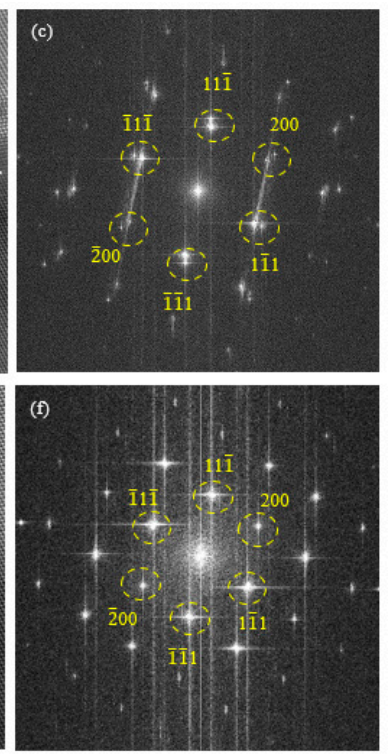

Figure 5. STEM and FFT patterns of the $\mathrm{HgCdTe} / \mathrm{CdTe}$ interface of Sample $1(\mathbf{a}-\mathbf{c})$ and Sample $2(\mathbf{d}-\mathbf{f})$. (a,d) STEM cross-sectional images of Sample 1 and Sample 2, which show the interface situations are different. (b,e) Magnified STEM of Sample 1 and Sample 2. The FFT patterns of (c) Sample 1 and (f) Sample 2 .

\subsection{Raman Spectra}

Figure 6 shows the Raman spectra of Sample 1 (a) and Sample 2 (b). Raman spectroscopy, which originates from molecular vibration and rotation, is sensitive to the structure, composition and defects of the material, and thus provides one of the main means of characterization for studying the lattice integrity, composition uniformity and crystal quality of the material. For the Sample 1, two defect peaks and three phonon features were observed which are located at $81,107,128,140$ and $153 \mathrm{~cm}^{-1}$. The peaks at $128 \mathrm{~cm}^{-1}$ and $140 \mathrm{~cm}^{-1}$ can be assigned to the HgTe-like transverse optical (TO) mode and longitudinal optical (LO) mode, the peak at $153 \mathrm{~cm}^{-1}$ can be assigned to the CdTe-like TO and LO mode. For the Sample 2, one defect peak and two phonon features were observed which are located at 87,116 , and $137 \mathrm{~cm}^{-1}$, and the peaks at 116 and $137 \mathrm{~cm}^{-1}$ is the HgTe-like TO and LO mode. The Raman peak positions and FWHMs of Sample 1 and Sample 2 as shown in Table 3. The peaks at 81 and $87 \mathrm{~cm}^{-1}$ are named $D_{1}$, the peak at $107 \mathrm{~cm}^{-1}$ is named $\mathrm{D}_{2}$. Compared with Sample 2, the $\mathrm{TO}_{1}$ and $\mathrm{LO}_{1}$ peaks of Sample 1 shift to higher frequencies. Due to the stacking faults in Sample 1, the lattice distortion is caused, which leads to compressive stress inside the crystal.
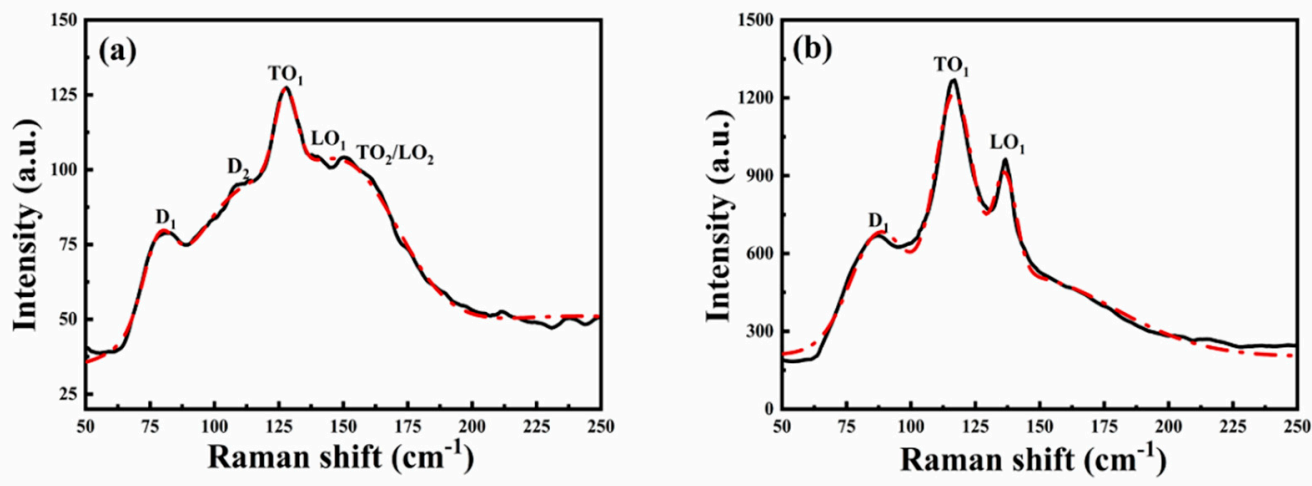

Figure 6. Raman diagrams of Sample 1 (a) and Sample 2 (b). 
Table 3. Raman peak positions and FWHMs of Sample 1, Sample 2.

\begin{tabular}{|c|c|c|c|c|c|c|c|}
\hline Sample & $\mathrm{D}_{1}\left(\mathrm{~cm}^{-1}\right)$ & $\mathrm{D}_{2}\left(\mathrm{~cm}^{-1}\right)$ & $\begin{array}{l}\text { HgTe-Like } \\
\text { TO }_{1}\left(\mathrm{~cm}^{-1}\right)\end{array}$ & $\begin{array}{c}\mathrm{TO}_{1} \mathrm{FWHM} \\
\left(\mathrm{cm}^{-1}\right)\end{array}$ & $\begin{array}{l}\text { HgTe-Like } \\
\text { LO }_{1}\left(\mathrm{~cm}^{-1}\right)\end{array}$ & $\begin{array}{c}\mathrm{LO}_{1} \mathrm{FWHM} \\
\left(\mathrm{cm}^{-1}\right)\end{array}$ & $\begin{array}{c}\text { CdTe-Like } \\
\mathrm{TO}_{2} / \mathrm{LO}_{2}\left(\mathrm{~cm}^{-1}\right)\end{array}$ \\
\hline Sample 1 & 81 & 107 & 128 & 10.5 & 140 & - & 153 \\
\hline Sample 2 & 87 & - & 116 & 16.2 & 137 & 8.9 & - \\
\hline
\end{tabular}

As can be seen in Figure 6, $D_{1}$ defect peaks are found in both Sample 1 and Sample 2, which is due to the lattice disorder of $\mathrm{HgCdTe}$ grown by MBE. Mazur [20] studied this peak and found that the peak disappeared after the annealing process. Annealing can improve the crystal quality and make the crystal lattice from disorder to order. The $\mathrm{D}_{2}$ defect peak in Sample 1 is related to Hg-deficiency, which has been previously explained in another article [21]. There are many Hg-deficiencies on the surface of Sample 1 (as shown in Figure 3a), so the peak related to Hg-deficiency can be detected by Raman spectra. It has been reported that low temperature annealing in $\mathrm{Hg}$ atmosphere can reduce $\mathrm{Hg}$ vacancies and thus eliminate the Raman peak of $\mathrm{Hg}$ vacancies. Moreover, there is a large number of Hg-deficiency in Sample 1, which causes lattice distortion. The shape and position of characteristic Raman peaks LO and TO are mainly related to lattice defects [22]. Therefore, the $\mathrm{LO}_{1}$ peaks of Sample 1 are not obvious, while the $\mathrm{LO}_{1}$ peaks of Sample 2 are obvious and symmetrical. As shown in Figure 6a, there are $\mathrm{TO}_{2} / \mathrm{LO}_{2}$ peaks for Sample 1, which may cause by the uneven surface composition caused by the $\mathrm{Hg}$ deficiencies.

The FWHM of the $\mathrm{TO}_{1}$ for $\mathrm{HgCdTe}$ Sample 1 and Sample 2 are $10.5 \mathrm{~cm}^{-1}$ and $16.2 \mathrm{~cm}^{-1}$, and the FWHM of $\mathrm{LO}_{1}$ for Sample 2 is $8.9 \mathrm{~cm}^{-1}$. Through the comparison of the Raman diagram, it can be seen that Sample 2 (grown at $151^{\circ} \mathrm{C}$ ) has fewer defects than Sample 1 (grown at $155^{\circ} \mathrm{C}$ ), and the crystal quality is better. The FWHMs of $\mathrm{TO}_{1}$ and $\mathrm{LO}_{1}$ of Sample 2 are similar to those of the other article [23].

\subsection{Photoluminescence Spectroscopy}

The photoluminescence (PL) is a nondestructive optical test method widely used to detect impurities, defects, and crystal quality of materials. Sample 1 and Sample 2 were subjected to variable temperature PL measurement and excited with a laser power of $100 \mathrm{~mW}$, power density is $1.4 \times 10^{5} \mathrm{~mW} / \mathrm{cm}^{2}$. Figure $7 \mathrm{a}, \mathrm{b}$ shows the normalized PL diagram of the samples. It can be seen from Figure $7 a, b$ the PL peaks of the two samples blue-shifted and broadened with the increase in temperature, which is caused by the increase in $\mathrm{Cd}$ concentration. Both Sample 1 and Sample 2 have double peaks above $160 \mathrm{~K}$, which is caused by $\mathrm{CO}_{2} / \mathrm{H}_{2} \mathrm{O}$ in the environment [24]. The asymmetry of the PL spectrum is mainly due to the thermal distribution effect of the high-temperature carrier. At $9 \mathrm{~K}$, the FWHMs of Sample 1 and Sample 2 are 25.2 and $19.2 \mathrm{meV}$, respectively. The FWHMs of the PL of the Sample 2 are smaller than that of the Sample 1, which proves that the crystal quality of the Sample 2 is better than that of the Sample 1.
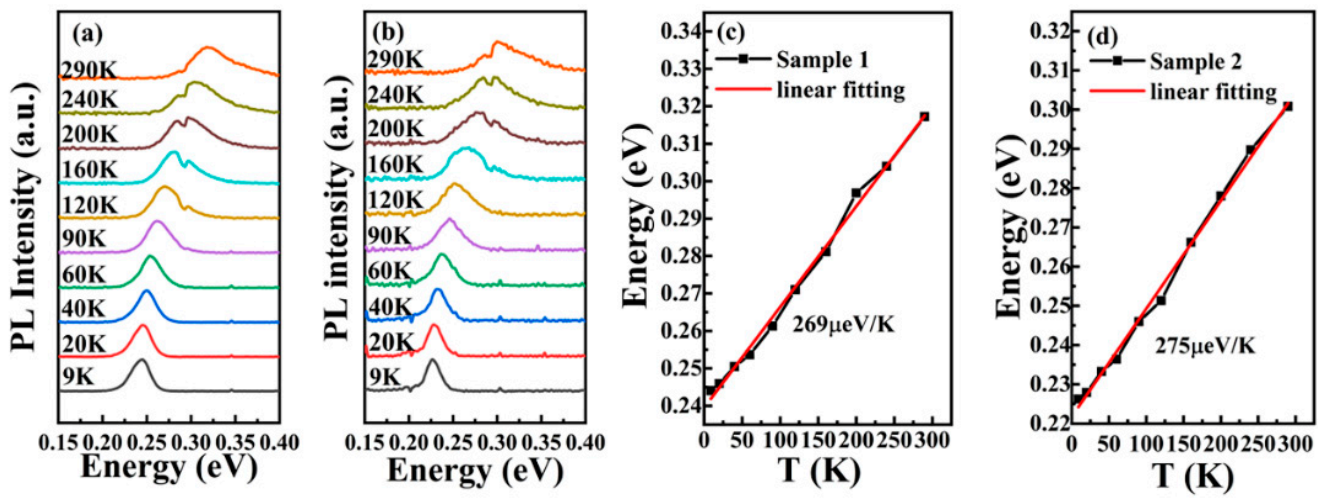

Figure 7. The variable temperature PL of Sample 1 (a) and Sample 2 (b). The temperature dependence of the main PL peak of Sample 1 (c) and Sample 2 (d). 
Figure 7c,d shows the temperature dependence of the main PL peak of the HgCdTe film. The solid line represents the relationship between $\mathrm{E}_{\mathrm{g}}$ and temperature, while the data points represent test results. It can be seen from Figure $7 c, d$ that the values of $E_{g}{ }^{P L}$ for Sample 1 and Sample 2 have a similar temperature coefficient $(269 \mu \mathrm{eV} / \mathrm{K}$ and $275 \mu \mathrm{eV} / \mathrm{K}$, respectively), which are higher than the $E_{\mathrm{g}}$ temperature coefficient of $195 \mu \mathrm{eV} / \mathrm{K}$ according to the calculation results of the formula 1. H.Wang et al. [25] have reported the similar results for LPE growth $\mathrm{HgCdTe}$ film. As PL transitions of HgCdTe film related to the excitonic effect and the band-tail of states at low temperatures present more evidently, and make the $E_{g}{ }^{P L}$ red-shift as compared to the $E_{g}$. Thus, the coefficient of $E_{g}{ }^{P L}$ and $E_{g}$ of the $\mathrm{HgCdTe}$ film are quite different. From the variable temperature spectra, the activation energy $\Delta \mathrm{E}$ of the HgCdTe Sample 1 and Sample 2 are $1.30 \pm 0.14$ and $0.75 \pm 0.16 \mathrm{meV}$, respectively. Due to the absorption of the peak by $\mathrm{CO}_{2}$, the accurate result of the correlation of the peak intensity to $1 / \mathrm{T}$ is affected. The reason for small activation energy of the films is still under study.

As shown above, the $\mathrm{HgCdTe}$ film grown at $155^{\circ} \mathrm{C}$ has many $\mathrm{Hg}$-deficiencies and poor crystal quality. Additionally, there is micro-twinning along the [111] orientation at the $\mathrm{HgCdTe} / \mathrm{CdTe}$ interface. The formation of micro-twinning caused by stacking faults formed by the Te-rich and the lattice mismatch between CdTe and HgCdTe. The HgCdTe film grown at $151{ }^{\circ} \mathrm{C}$ does not have a large number of defects and stacking faults at the $\mathrm{HgCdTe} / \mathrm{CdTe}$ interface, and the crystal quality is better that of $\mathrm{HgCdTe}$ film grown at $155^{\circ} \mathrm{C}$. This shows that temperature has a significant effect on the crystal quality of $\mathrm{HgCdTe}$ thin films.

\section{Conclusions}

In this work, the single-crystal HgCdTe (211) films were successfully grown on GaAs (211) B substrates by MBE, and the effects of growth temperature on the crystal quality of $\mathrm{HgCdTe} / \mathrm{CdTe}$ interface were studied. The $\mathrm{HgCdTe}$ film grown at $151^{\circ} \mathrm{C}$ has high crystal quality, the interface is flat and there are no micro-twins. The crystal quality of the $\mathrm{HgCdTe}$ grown at $155^{\circ} \mathrm{C}$ is poor, and there are many defects and micro-twins at the interface, which are caused by the high temperature-induced Te-rich defects. Raman peaks of HgCdTe with growth temperatures of $155^{\circ} \mathrm{C}$ also demonstrate that the higher temperature would cause the lattice disorder of $\mathrm{HgCdTe} / \mathrm{CdTe}$ interfaces. The research results demonstrate that the temperature significantly affects the crystal quality and optical properties of $\mathrm{HgCdTe}$ films.

Author Contributions: Data curation, X.-F.Q., S.-X.Z., J.Z., Y.-C.Z., C.D. and S.-C.H.; Formal analysis, X.-F.Q. and P.-P.C.; Funding acquisition, P.-P.C.; Methodology, X.-F.Q., S.-X.Z., J.Z., Y.W., S.-C.H. and P.-P.C.; Supervision, Y.W. and P.-P.C.; Validation, P.-P.C.; Writing—original draft, X.-F.Q.; Writingreview \& editing, X.-F.Q. and P.-P.C. All authors have read and agreed to the published version of the manuscript.

Funding: This work was supported by the National Nature Science Foundation of China (Grant Nos. $11634009,11991060,11991063,11774016)$.

Institutional Review Board Statement: Not applicable.

Informed Consent Statement: Not applicable.

Data Availability Statement: All data are fully available without restriction. The datasets used and/or analyzed during the current study are available from the corresponding author on reasonable request.

Acknowledgments: We acknowledge the financial support from the National Nature Science Foundation of China (Grant Nos. 11634009, 11991060, 11991063, 11774016) is gratefully acknowledged.

Conflicts of Interest: The authors declare no conflict of interest. 


\section{References}

1. Rogalski, A.; Razeghi, M. Narrow-gap semiconductor photodiodes. In Proceedings of the Photodetectors: Materials and Devices III, San Jose, CA, USA, 8 April 1998; Volume 3287, pp. 2-13.

2. Hoang, A.M.; Dehzangi, A.; Adhikary, S.; Razeghi, M. High performance bias-selectable three-color Short-wave/Mid-wave/Longwave Infrared Photodetectors based on Type-II InAs/GaSb/AlSb superlattices. Sci. Rep. 2016, 6, 24144. [CrossRef]

3. Lei, W.; Antoszewski, J.; Faraone, L. Progress, challenges, and opportunities for HgCdTe infrared materials and detectors. Appl. Phys. Rev. 2015, 2, 41303. [CrossRef]

4. Rogalski, A.; Martyniuk, P.; Kopytko, M. InAs/GaSb type-II superlattice infrared detectors: Future prospect. Appl. Phys. Rev. 2017, 4, 31304. [CrossRef]

5. Lü, Y.Q.; Lu, X.; Lu, Z.X.; Li, M. Review of Antimonide Infrared Detector Development at Home and Abroad. Aero Weapon. 2020, 27, 1-12.

6. Destéfanis, G.; Tribolet, P.; Vuillermet, M.; Lanfrey, D.B. MCT IR detectors in France. In Proceedings of the Infrared Technology and Applications XXXVII, Orlando, FL, USA, 20 May 2011; Volume 8012, p. 801235.

7. Kinch, M.A. High-operating-temperature (HOT) detector requirements. Mater. Infrared Detect. 2001, 4454, 168-179.

8. Perrais, G.; Gravrand, O.; Baylet, J.; Destefanis, G.; Rothman, J. Gain and dark current characteristics of planar HgCdTe avalanche photo diodes. J. Electron. Mater. 2007, 36, 963-970. [CrossRef]

9. Rothman, J.; Perrais, G.; Destefanis, G.; Baylet, J.; Castelein, P.; Chamonal, J.-P. High performance characteristics in pin MW HgCdTe e-APDs. In Proceedings of the Infrared Technology and Applications XXXIII, Orlando, FL, USA, 14 May 2007; Volume 6542, p. 654219.

10. Lei, W.; Gu, R.; Antoszewski, J.; Dell, J.; Faraone, L. GaSb: A new alternative substrate for epitaxial growth of HgCdTe. J. Electron. Mater. 2014, 43, 2788-2794. [CrossRef]

11. Zhao, J.; Yang, Y.L.; Li, Y.H.; Song, L.Y.; Ji, R.B. The Study of MBE Molecule Beam Based on the Ensemble Theory. Infrared Technol. 2006, 28, 466-469.

12. Chang, Y.; Zhao, J.; Abad, H.; Grein, C.H.; Sivananthan, S.; Aoki, T.; Smith, D.J. Performance and reproducibility enhancement of $\mathrm{HgCdTe}$ molecular beam epitaxy growth on CdZnTe substrates using interfacial HgTe/CdTe superlattice layers. Appl. Phys. Lett. 2005, 86, 131924. [CrossRef]

13. He, L.; Wu, Y.; Chen, L. Composition control and surface defects of MBE-grown HgCdTe. J. Cryst. Growth 2001, 227, 677-682. [CrossRef]

14. Lei, W.; Ren, Y.L.; Madni, I.; Faraone, L. Low dislocation density MBE process for CdTe-on-GaSb as an alternative substrate for HgCdTe growth. Infrared Phys. Technol. 2018, 92, 96-102. [CrossRef]

15. Chu, J.H. Narrow Band Gap Semiconductor Physics; Science Press: Beijing, China, 2005; p. 933.

16. Benson, J.D.; Almeida, L.A.; Carmody, M.W.; Edwall, D.D.; Markunas, J.K.; Jacobs, R.N.; Martinka, M.; Lee, U. Surface Structure of Molecular Beam Epitaxy (211) B HgCdTe. J. Electron. Mater. 2007, 36, 949-957. [CrossRef]

17. Tejedor, P.; Šmilauer, P.; Roberts, C.; Joyce, B.A. Surface-morphology evolution during unstable homoepitaxial growth of GaAs (110). Phys. Rev. B 1999, 59, 2341-2345. [CrossRef]

18. Lei, W.; Gu, R.; Antoszewski, J.; Dell, J.; Neusser, G.; Sieger, M.; Mizaikoff, B.; Faraone, L. MBE growth of mid-wave infrared HgCdTe layers on GaSb alternative substrates. J. Electron. Mater. 2015, 44, 3180-3187. [CrossRef]

19. Yang, B.; Xin, Y.; Rujirawat, S.; Browning, N.D.; Sivananthan, S. Molecular beam epitaxial growth and structural properties of HgCdTe layers on CdTe (211) B/Si (211) substrates. J. Appl. Phys. 2000, 88, 115-119. [CrossRef]

20. Mazur, Y.I.; Kriven, S.I.; Tarasov, G.G.; Shevchenko, N.V. Vibrational modes activated by structural disorder in FIR transmission of $\mathrm{Hg} 1_{-\mathrm{x}-\mathrm{y}} \mathrm{Cd}_{\mathrm{x}} \mathrm{Mn}_{\mathrm{y}}$ Te. Semicond. Sci. Technol. 1993, 8, 1187. [CrossRef]

21. Huang, S.; Xia, C.; Zhang, P. Defect analysis in mercury cadmium telluride by Raman scattering. Spectrosc. Spectr. Anal. 2001, $21,492-494$.

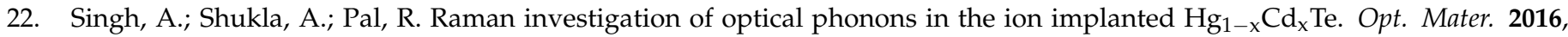
57, 34-38. [CrossRef]

23. Singh, A.; Pal, R.; Dhar, V.; Pant, S.C. Raman spectroscopic study of HgCdTe epilayers for infrared detector array fabrication. Infrared Phys. Technol. 2011, 54, 44-47. [CrossRef]

24. Zhang, X.; Shao, J.; Chen, L.; Lu, X.; Guo, S.; He, L.; Chu, J. Infrared photoluminescence of arsenic-doped HgCdTe in a wide temperature range of up to $290 \mathrm{~K}$. J. Appl. Phys. 2011, 110, 43503. [CrossRef]

25. Wang, H.; Hong, J.; Yue, F.; Jing, C.; Chu, J. Optical homogeneity analysis of $\mathrm{Hg}_{1-\mathrm{x}} \mathrm{Cd}_{\mathrm{x}} \mathrm{Te}$ epitaxial layers: How to circumvent the influence of impurity absorption bands? Infrared Phys. Technol. 2017, 82, 1-7. [CrossRef] 